\title{
A BAC-based physical map of Brachypodium distachyon and its comparative analysis with rice and wheat Yong Q Gu${ }^{1}$, Yaqin $\mathrm{Ma}^{2}$, Naxin Huo ${ }^{1,2}$, John P Vogel ${ }^{1}$, Frank M You ${ }^{1,2}$, Gerard R Lazo1, William M Nelson³, Carol Soderlund ${ }^{3}$, Jan Dvorak², Olin D Anderson ${ }^{1}$ and Ming-Cheng Luo*2
}

\author{
Address: ${ }^{1}$ Genomics and Gene Discovery Research Unit, USDA-ARS, Western Regional Research Center, 800 Buchanan Street, Albany, CA 94710 , \\ USA, ${ }^{2}$ Department of Plant Sciences, University of California, Davis, CA 95616, USA and ${ }^{3}$ BIO5 Institute, University of Arizona, Tucson, AZ 85721, \\ USA \\ Email: Yong Q Gu - Yong.Gu@ars.usda.gov; Yaqin Ma - ama@ucdavis.edu; Naxin Huo - naxin.huo@ars.usda.gov; \\ John P Vogel - John.Vogel@ars.usda.gov; Frank M You - Frank.You@ars.usda.gov; Gerard R Lazo - Gerard.Lazo@ars.usda.gov; \\ William M Nelson - will@agcol.arizona.edu; Carol Soderlund - Cari@agcol.arizona.edu; Jan Dvorak - jdvorak@ucdavis.edu; \\ Olin D Anderson - Olin.Anderson@ars.udsa.gov; Ming-Cheng Luo* - mcluo@ucdavis.edu \\ * Corresponding author
}

BMC Genomics 2009, 10:496 doi:10.1186/147|-2164-10-496

This article is available from: http://www.biomedcentral.com/I47I-2/64/I0/496

(c) 2009 Gu et al; licensee BioMed Central Ltd.

This is an Open Access article distributed under the terms of the Creative Commons Attribution License (http://creativecommons.org/licenses/by/2.0), which permits unrestricted use, distribution, and reproduction in any medium, provided the original work is properly cited.

\begin{abstract}
Background: Brachypodium distachyon (Brachypodium) has been recognized as a new model species for comparative and functional genomics of cereal and bioenergy crops because it possesses many biological attributes desirable in a model, such as a small genome size, short stature, self-pollinating habit, and short generation cycle. To maximize the utility of Brachypodium as a model for basic and applied research it is necessary to develop genomic resources for it. A BAC-based physical map is one of them. A physical map will facilitate analysis of genome structure, comparative genomics, and assembly of the entire genome sequence.

Results: A total of 67,I5I Brachypodium BAC clones were fingerprinted with the SNaPshot HICF fingerprinting method and a genome-wide physical map of the Brachypodium genome was constructed. The map consisted of 671 contigs and 2,16I clones remained as singletons. The contigs and singletons spanned $414 \mathrm{Mb}$. A total of 13,970 gene-related sequences were detected in the BAC end sequences (BES). These gene tags aligned 345 contigs with $336 \mathrm{Mb}$ of rice genome sequence, showing that Brachypodium and rice genomes are generally highly colinear. Divergent regions were mainly in the rice centromeric regions. A dot-plot of Brachypodium contigs against the rice genome sequences revealed remnants of the whole-genome duplication caused by paleotetraploidy, which were previously found in rice and sorghum. Brachypodium contigs were anchored to the wheat deletion bin maps with the BES gene-tags, opening the door to Brachypodium-Triticeae comparative genomics.
\end{abstract}

Conclusion: The construction of the Brachypodium physical map, and its comparison with the rice genome sequence demonstrated the utility of the SNaPshot-HICF method in the construction of BAC-based physical maps. The map represents an important genomic resource for the completion of Brachypodium genome sequence and grass comparative genomics. A draft of the physical map and its comparisons with rice and wheat are available at http://phymap.ucdavis.edu/brachypodium/. 


\section{Background}

Model systems play an important role in studies of genome structure and evolution, and are invaluable in gene isolation and functional characterization. The application of model systems toward the study of both basic and applied problems in plant biology has become routine. The model dicot Arabidopsis thaliana has been used in studies ranging from nutrient uptake and metabolism to plant-pathogen interactions. Unfortunately, due to its distant relationship to monocots, Arabidopsis is not an ideal model for grasses. Rice is being currently used as a grass model [1], but its primary adaptation to semi-aquatic, subtropical environments limits its usefulness. The large sizes of rice plants and long generation time make experiments requiring large numbers of plants grown under controlled conditions costly. It is also challenging to grow rice under the conditions prevailing in greenhouses in northern climates.

Brachypodium distachyon has numerous attributes expected to find in a genetic model and interest in using it as a model system for wheat and other temperate grasses is growing rapidly [2-8]. Diploid B. distachyon is closely related to the Triticeae $[9,10]$ but in contrast to the Triticeae, it possesses a very small genome $(\mathrm{x}=5)$ of approximately $355 \mathrm{Mb}[9,11]$. The recent release of $8 \times \mathrm{B}$. distachyon genome sequence showed that the genome is $271 \mathrm{Mb}$ in size (assembled sequences, http://www.brach ypodium.org). It is a small temperate grass with simple growth requirements, short generation time, and self-pollinating habit $[2,6,7,9]$. Highly efficient transformation of B. distachyon via Agrobacterium tumefaciens has been developed, which will facilitate its functional genomics and biotechnological applications [12-14]. These characteristics make $B$. distachyon superbly suitable for both functional and comparative genomic research.

Several genomic regions of $B$. distachyon and B. sylvaticum, a close relative of $B$. distachyon with a larger genome, have been compared with wheat and rice. In general, good colinearity was observed reflecting general conservation of synteny across the grass family [15-19]. To foster the development of $B$. distachyon as a grass model and coordinate the development of its genomics resources, the International Brachypodium Initiative was formed http:// www.brachypodium.org. The Initiative placed a high priority on the development of a global physical map of diploid $B$. distachyon composed of large genomic fragments cloned in a bacterial artificial chromosome vector (BAC) http://www.brachypodium.org/node/8. A high resolution BAC-based physical map has many genomics applications including analyzing genome structure, conducting genome-wide comparisons, and facilitating the assembly of $B$. distachyon genome sequence.
The development of a Brachypodium BAC-based physical map is reported here. Also reported is a global comparison of the map with rice genome sequence [1] and wheat deletion bin maps [20] with the goal to obtain a clearer picture of $B$. distachyon genome structure and evolutionary history and their relationships to those of rice and wheat.

\section{Results and Discussion \\ $B A C$ source, fingerprinting, and contig assembly}

A total of 67,151 clones of HindIII and BamHI BAC libraries developed from the diploid $B$. distachyon accession $\mathrm{Bd} 21$ [21] were fingerprinted using the SNaPshot HICF BAC fingerprinting method $[22,23]$. To generate more information about each clone, a GS1200Liz size standard, which allows sizing of restriction fragments up to 1,000 bp (Figure 1A), was used. The use of GS1200Liz necessitated using the $50-\mathrm{cm}$ capillary array for the ABI $3730 \mathrm{XL}$, instead of the standard $36-\mathrm{cm}$ capillary array that is used for electrophoresis of fragments ranging from 50 bp to 500 bp $[22,24,25]$. Large-size fragments are less frequent than small-size fragments in the SNaPshot HICF profiles (Figure 1B), and are more valuable in contig assembly because they are less likely to be shared by chance [22]. Since more large fragments could be called using the GS1200Liz as size standard, fragments with size less than $100 \mathrm{bp}$ were not used for contig assembly in this study.

Cross-contamination and low quality fingerprinting data interfere with accurate contig assembly [24]. Contaminated clones, empty clones, small insert clones, and clones with fingerprints below specified quality threshold were eliminated with the GenoProfiler program [26]. Of the 67,151 fingerprinted clones, 52,343 clones (78\%) were suitable for contig assembly. An average fingerprint had 79.4 restriction fragments in this population of fingerprints. Since the average insert size was $100 \mathrm{~kb}$ [21], there was on the average a restriction fragment every 1.26 $\mathrm{kb}$.

The 52,343 fingerprints representing $14 \times B$. distachyon genome equivalents were used for an initial automated contig assembly using the FPC software [27]. The initial assembly was performed at a relatively high stringency (1 $\times 10^{-45}$ ) to minimize faulty contig assembly of clones from unrelated regions of the genome. The "DQer" function was used to dissemble contigs containing more than 10\% questionable (Q) clones. The "End to End" FPC function was then repeatedly employed to merge contigs with successively less stringent Sulston score cutoff values $[24,25,28]$. In the end, the FPC assembly resulted in 648 contigs containing a total of 50,182 BAC clones. In this "Phase I" physical map, 177 contigs had more than 100 clones each, 73 contigs had 50 - 99 clones each, 72 contigs had 10 - 49 clones, and the rest had 9 clones or less. A total of 2,161 singletons remained. The cumulative, contigu- 


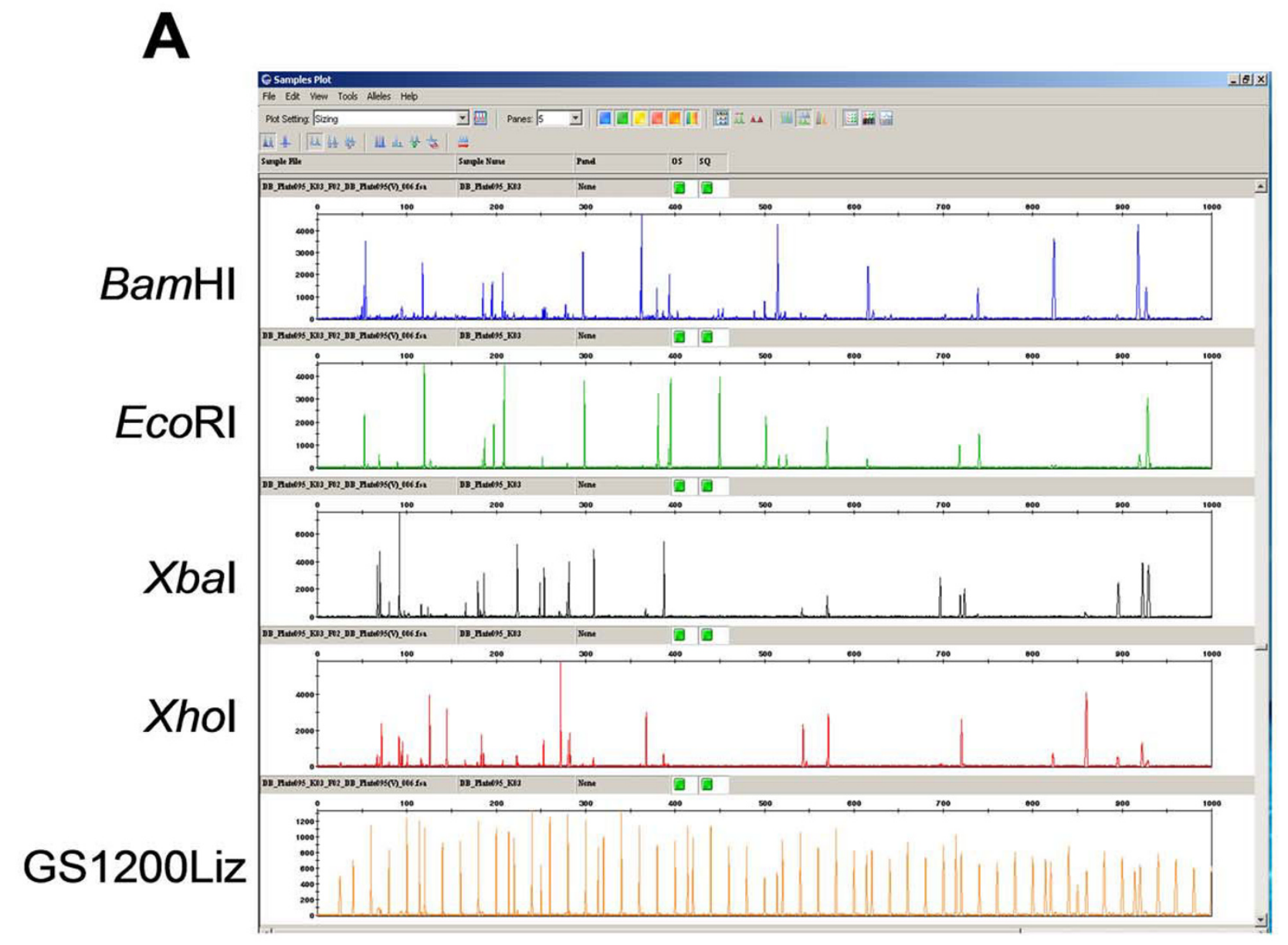

B

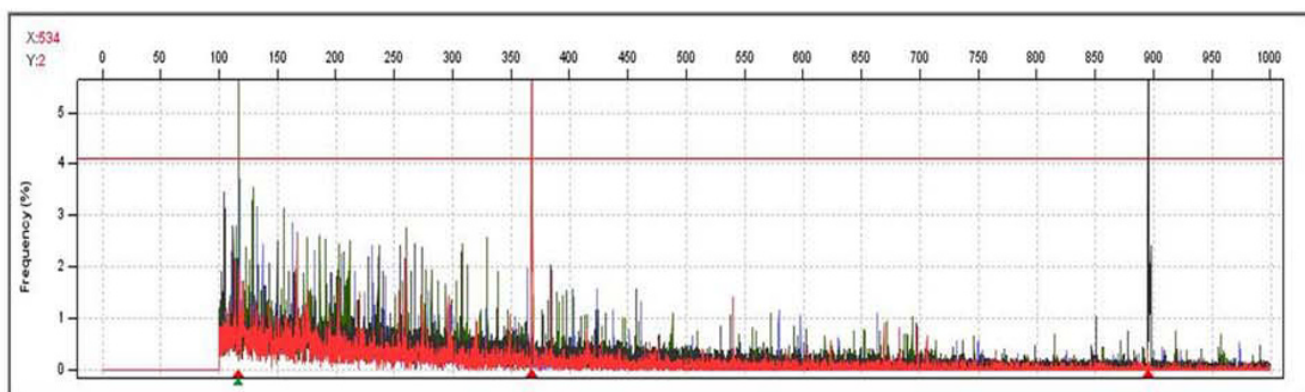

\section{Figure I}

Fragment sizing with ABI 3730xI and frequency distribution of fragment sizes using GS I 200Liz size standard. Figure IA shows an example of fingerprinting profile of a digested BAC clone using GSI200Liz as a size standard. The fingerprinting of each BAC involved digestion with five restricted enzymes and labeling with four fluorescent dyes as described previously [22]. The size for each fragment was calculated based on co-migration of size standard in the capillary. Figure IB shows the frequency of fragments with different sizes in 14,23I fingerprinted Brachypodium BAC clones. Large peaks represent vector fragments that appear in high frequencies. The red line defines the threshold for high frequency fragments derived from BAC inserts. Fragments with a frequency above the threshold were removed prior to contig assembly due to their likely origin from repetitive sequences. 
ous, non-redundant fragment count across all contigs was equivalent to approximately $410 \mathrm{Mb}$, which was $15.5 \%$ more than the estimated size of $B$. distachyon genome (355 $\mathrm{Mb})[9,11]$; if the genome size of $271 \mathrm{Mb}$ based on the recent release of $8 \times$ genome sequence assembly http:// www.brachypodium.org is used, the fragment count would be equivalent to $51.3 \%$ more of the estimated genome size. This indicated that many contigs actually overlapped other contigs, but the overlaps were below contig joining threshold. Such overestimation has been reported in physical maps of other plant genomes [25,29].

\section{Editing of contigs by alignments with the rice genome sequence}

Integration of molecular markers into contigs is crucial for their anchoring on genetic maps and ultimate alignment of a physical map and genome sequence. This task can be accomplished by screening BAC libraries with pools of labeled probes derived from EST clones or mapped genetic markers or screening of multidimensional pools of BAC clones by PCR or highly parallel Illumina GoldenGate assays [30-33]. BAC end sequences (BESs), in addition to other genomic applications [34-37], can facilitate initial genome characterization $[3,28,34,35]$ and anchoring of contigs onto the genetic map. BESs are particularly useful for contig anchoring in small, gene-dense genomes. Their utility is diminished in large and complex genomes due to a low gene density. For example, in wheat, over $80 \%$ of the genome consists of repetitive DNA (reviewed in [38]). Akhunov et al. [39] reported that coding sequences accounted for only $5.8,4.5$, and $4.8 \%$ of BES in T. uratu, Ae. speltoides, and Ae. tauschii BAC libraries, respectively. A total of $38 \mathrm{Mb}$ of random $B$. distachyon genomic sequence was generated by sequencing 64,694 BAC ends from the two BAC libraries, representing $\sim 14.0 \%$ of the genome sequence on the basis of a genome size of $271 \mathrm{Mb}$ http://www.brachypodium.org. This was equivalent to one sequence tag every $4.2 \mathrm{~kb}$ (considering $271 \mathrm{Mb}$ of the genome size). A total of $25.3 \%$ of repeatmasked B. distachyon BESs had matches to the rice genome sequence $\left(E<10^{-25}\right)$. Among them, 13,970 also matched wheat ESTs [3]. Therefore, the integration of B. distachyon BES into the contigs immediately anchored a large number of contigs onto the rice genome sequence and wheat deletion maps (see discussion below).

BES of fingerprinted clones facilitated manual editing and contig assembly validation. This was based on the assumption that closely related grass genomes share extensive colinearity. The colinearity of contigs with the rice genome can be used to assess quality of SNaPshotbased BAC fingerprinting technology and contig assembly. Brachypodium contigs with BESs allowed for direct alignment of contigs with rice pseudomolecules; BLAT [40] was used for finding sequence similarities, which were then used by SyMAP (Synteny Mapping and Analysis Program [41]) for computing the synteny blocks and visualizing the results (Figure 2 and results below). These alignments were used to guide contig editing and disjoining, as it was inevitable that miss-assembled BAC contigs occurred due to a number of factors including chimeric clones and cross-contamination. In addition, contig merging was performed with successively increasing cutoffs (as high as $1 \times 10^{-14}$ ), so it was likely that some merging could result in false joining of two unrelated regions. We used alignments with the rice genome as reference to provide supporting evidence during disjoining problem contigs. During contig editing, when two merged contigs aligned to two different regions in the rice genome, the merge was rejected and the merged contigs were disjoined. The same strategy can be applied to miss-assembled contigs. When a contig is aligned to different rice genomic regions, the contig should be further evaluated to identify potential assembly problems. For example, in the initial assembly, Contig10 was aligned to two genomic blocks on rice chromosome 1 , separated by over $35 \mathrm{Mb}$ (Figure 2). It was found that the contig contained two clusters linked by two BAC clones, DB064D23 and DB064F23. These two clones reside near each other in a 96-well plate, indicating that cross-contamination may have occurred during fingerprinting process (inoculation or transfer) and probably resulted in two shared fingerprint profiles just below the predefined contamination threshold. Contig 10 was disjoined into two after removing the two clones during the contig editing process.

The integration of BES into contigs and manual editing of contigs using rice genome as a reference improved contig assembly by disjoining 23 contigs. The final assembly contained 671 contigs, which included BESs. This assembly is called "Phase II physical map" of the B. distachyon genome. Figure 3 shows an example of a contig in the Phase II physical map. The view of the complete set of $B$. distachyon contigs is available at http://phy map.ucdavis.edu/brachypodium/.

\section{Comparison of B. distachyon contigs with the rice genome} The alignment of contigs of the Phase II $B$. distachyon physical map to the rice genome sequence estimated the genome coverage. A total of 345 contigs (51.4\%) could be aligned to the rice genome sequence. They covered 336 $\mathrm{Mb}(88 \%)$ of the rice genome sequence (using $382 \mathrm{Mb}$ as $1 C$ rice genome size, [1]) and represented $88 \%$ of the total B. distachyon FPC map as measured by CB units. When only contigs with more than 10 clones were used, 331 out of $364(90.9 \%)$ could be aligned to the rice genome. Although 326 contigs could not be anchored, these contigs were generally small, and the total number of clones in them equaled to only $2,489(5.0 \%)$ out of the total 50,182 clones, indicating that only a small portion of the 


\section{Brachypodium Contig 10}

\section{Rice Chr1}

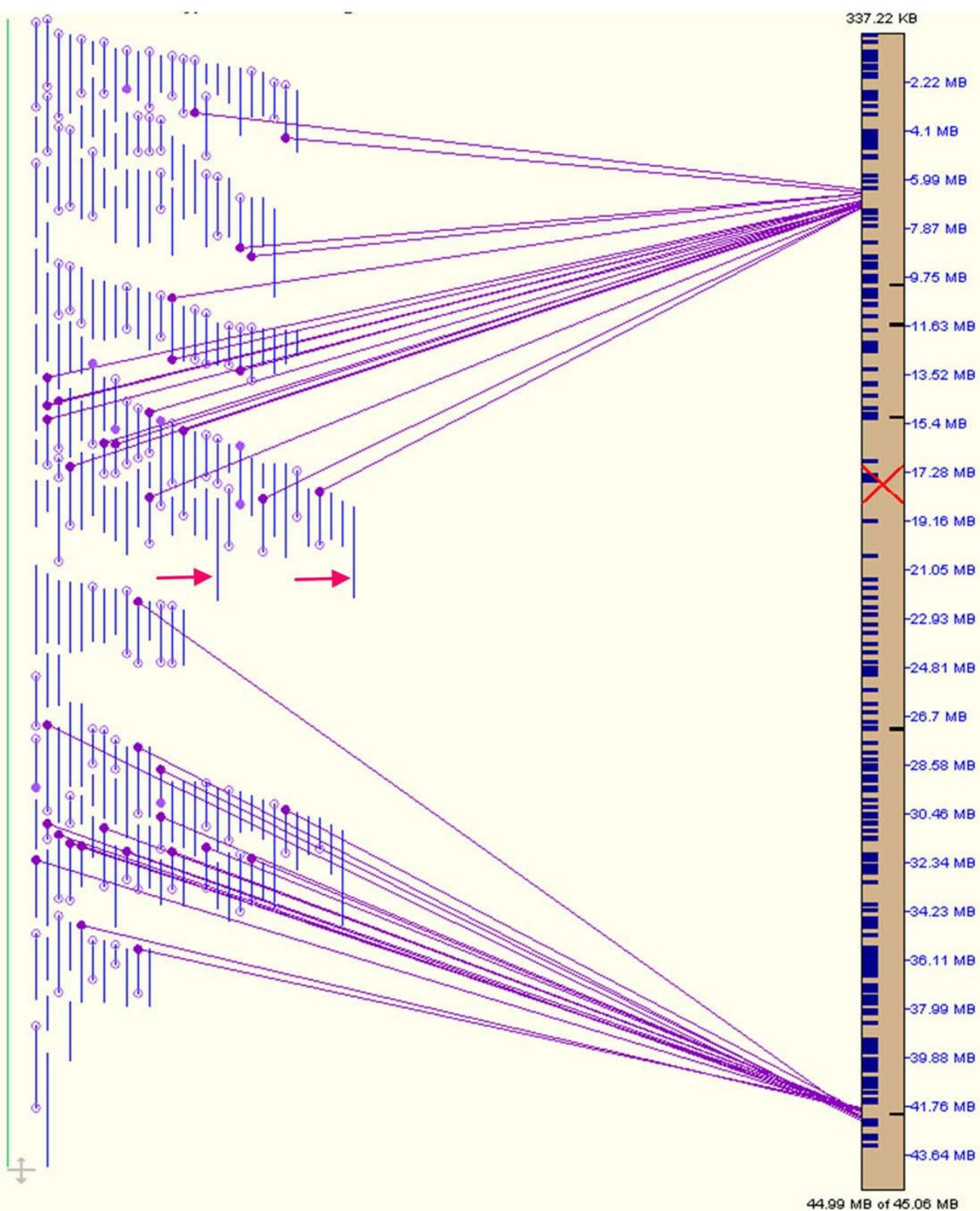

\section{Figure 2}

The SyMAP close-up view shows the false joining of contigs caused by clone contamination. Contig 10 from the Phase I assembly matched two rice regions that were separated by over $35 \mathrm{Mb}$ on rice chromosome I (Chr I). Solid vertical lines represent BAC clones. Dots at the ends of solid vertical lines represent BESs generated for the corresponding BAC clones. Empty dot represents BES with no significant match to the rice genome. The dots connected by lines indicate that the BESs have matches in the corresponding orthologous positions in the rice genome. Filled dots with no connecting lines indicate BESs with matches to rice sequences located in different regions of the rice genome. Two cross-contaminated clones that caused false joining of the two clusters are indicated by arrows (not part of the SyMAP display). 


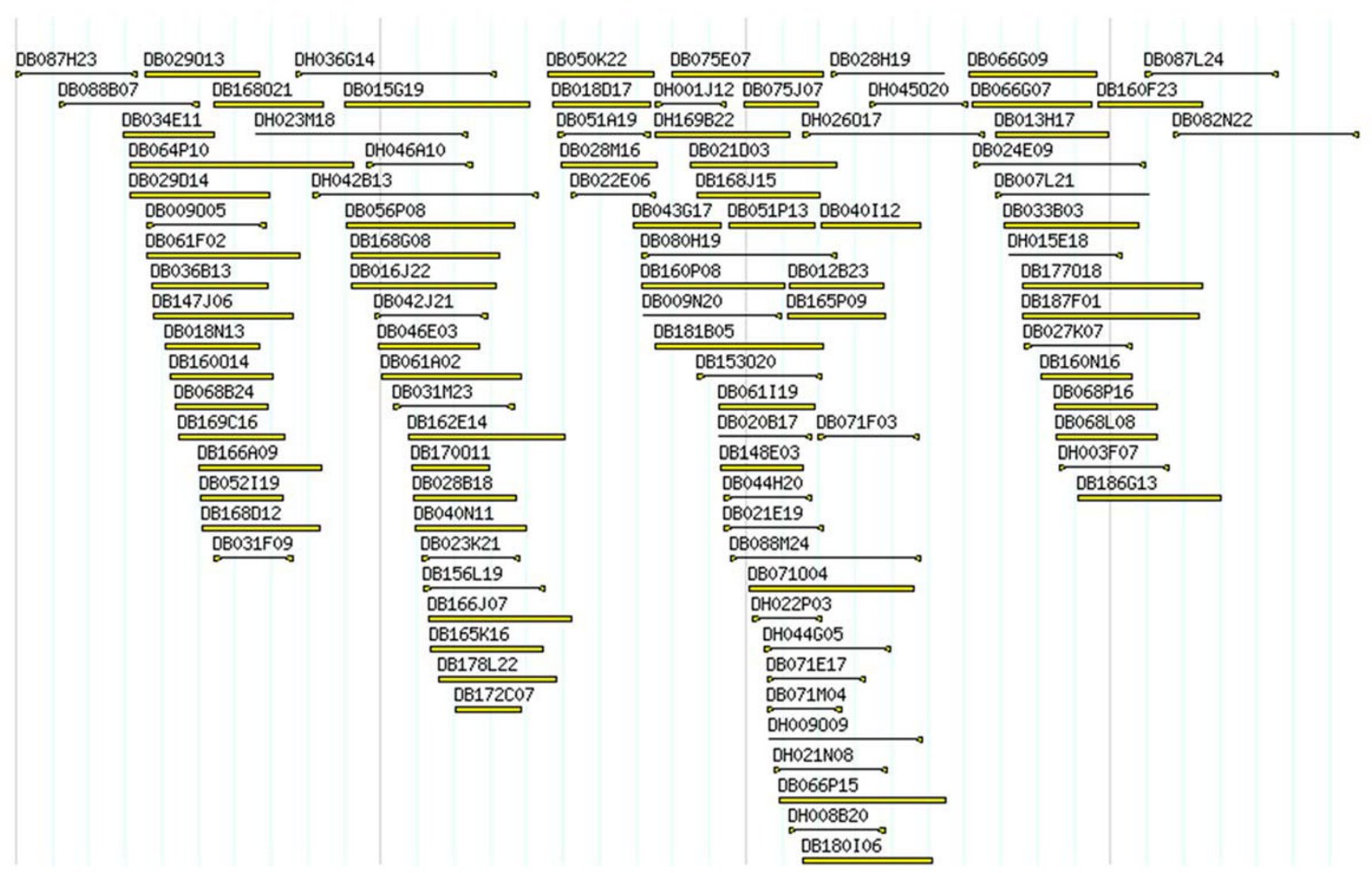

Figure 3

FPC contig view of a Brachypodium contig. Brachypodium Contig 133 is used as an example. Clones with solid black lines below the clone name represent those with BES. A triangle at both ends of the line indicated that both ends of the BAC clone were sequenced, while a triangle at one end indicates that BES is only present at that end. The clones prefixed with "DH" and "DB" were from B. distachyon Hindlll and BamHI BAC libraries, respectively.

clones could not been anchored onto the rice genome. The data suggested a general conservation of synteny between rice and $B$. distachyon genomes, which confirms previous conclusions made on the basis of sequencing a few B. sylvaticum BAC clones and their sequence comparisons with the orthologous regions in rice and wheat [15].

Ideally, B. distachyon contigs should be ordered using a high-resolution genetic map. Such a map was not available to us. However, the SyMAP alignment of the FPC contigs onto the sequenced rice chromosomes ordered many of the contigs into synteny blocks with putative chromosome assignments and showed extensive colinearity of contigs with the rice genome sequences. For instance, Contig 91 appeared to be highly colinear with a $7-\mathrm{Mb}$ region in rice chromosome 3 although several small local inversions could be identified (Figure 4). Out of 160 BESs in this contig homologous to the rice genome sequence, $117(73 \%)$ were homologous to sequences in this region on rice chromosome 3, while 53 were homologous with sequences in different regions of the rice genome, presumably representing non-colinear genes. Previous alignment of ten sequenced $B$. distachyon BAC clones with the orthologous rice regions also revealed a general synteny conservation between rice and $B$. distachyon [19]. It was found that $15 \%$ B. distachyon and $19 \%$ rice genes were not present in the corresponding orthologous regions [19]. The non-colinear genes reflect the divergence of the two genomes after the split from a common ancestor during phylogeny of the grass family [42].

A SyMap dot-plot (Figure 5A) shows correspondence of most $B$. distachyon contigs to specific regions of the rice genomic sequence. This is consistent with the estimation that the $B$. distachyon contigs cover $336 \mathrm{Mb}$ or $\sim 88 \%$ of the rice genome. A question remains whether or not these alignments provide evidence of general colinearity between rice and $B$. distachyon chromosomes. Although the $B$. distachyon contigs are not ordered, the following line of reasoning suggests that the $B$. distachyon and rice 


\section{Rice Chr3}

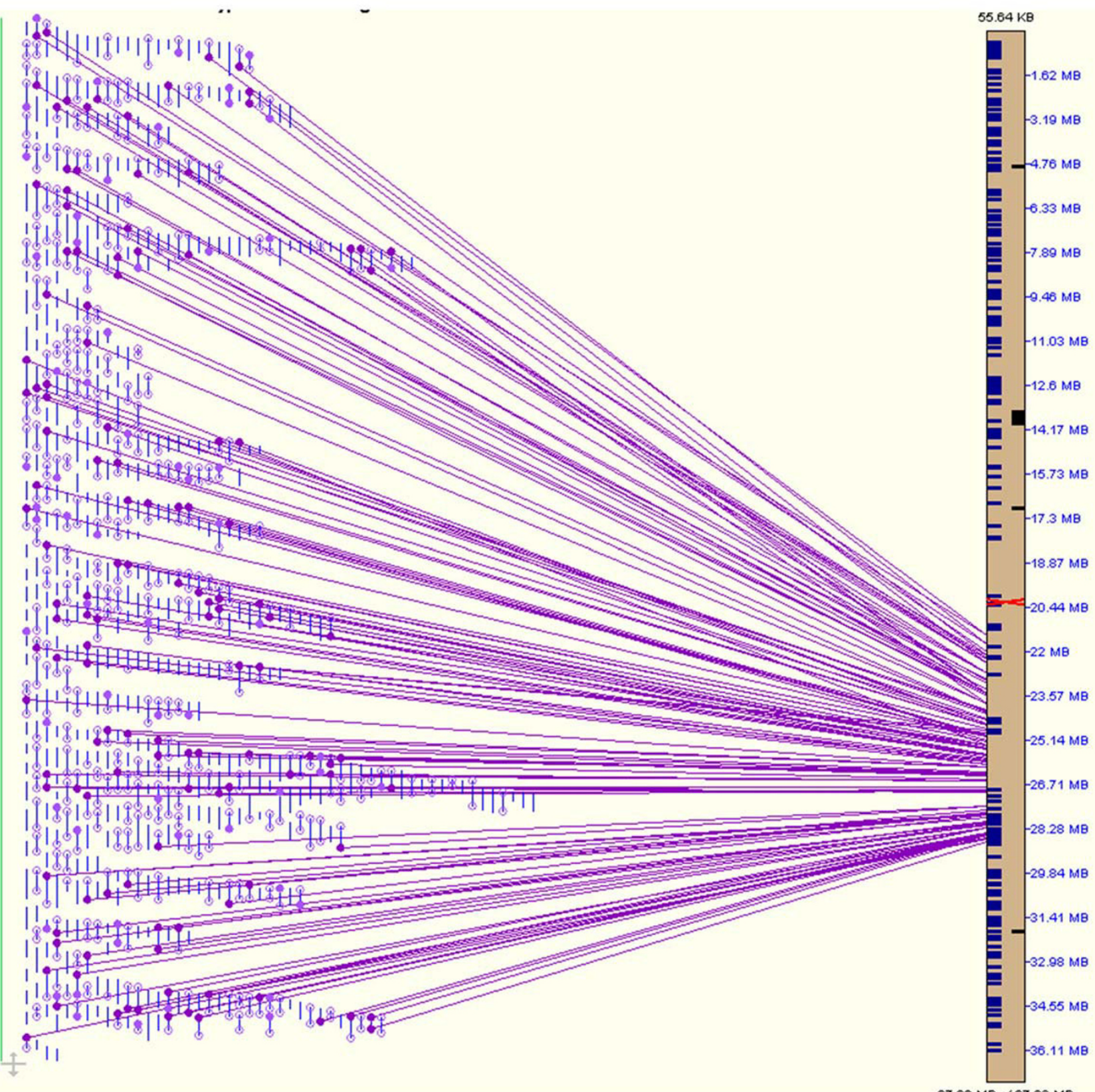

Figure 4

Colinearity of Brachypodium Contig 91 with a 7-Mb genomic region on rice chromosome 3 (Chr3). Contig 91 contains 678 BAC clones with a total of 549 BES. This contig aligned to a 7-Mb genomic region on rice Chr3 based on BLAT comparison. BESs that match rice sequence in the 7-Mb orthologous region were connected to the corresponding position with a solid line.

chromosomes are highly collinear. Differences in gene order between $B$. distachyon and rice due to inversions or translocations would be detected in B. distachyon BAC contigs as breaks in co-linearity not associated with week joins http://phymap.ucdavis.edu/brachypodium. Four- teen such genuine breaks in colinearity are expected between the two genomes reflecting the difference in chromosome number; $x=12$ in rice to $x=5$ in $B$. distachyon. Of the 364 contigs having more than 10 clones (see above), 33 may have such colinearity breaks. If 14 are due 


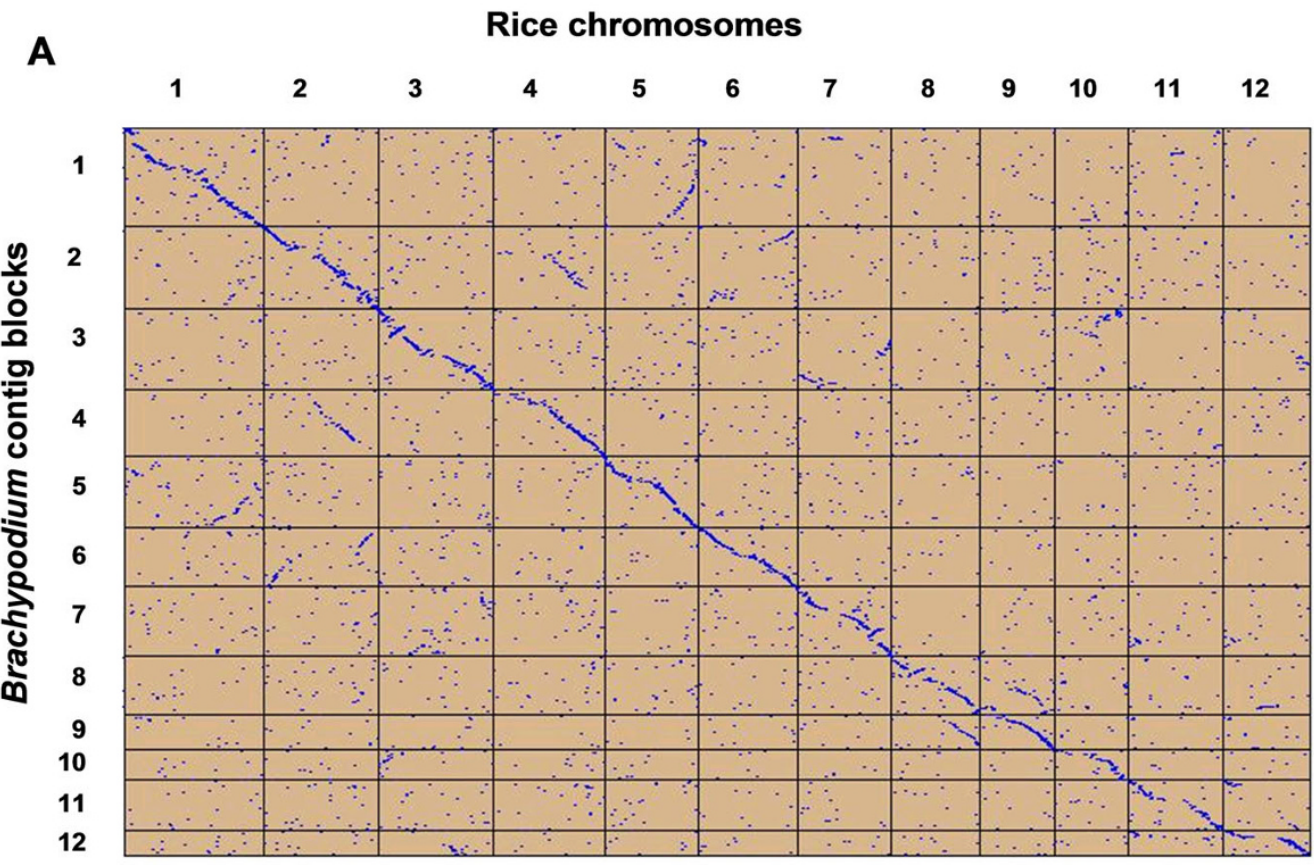

B

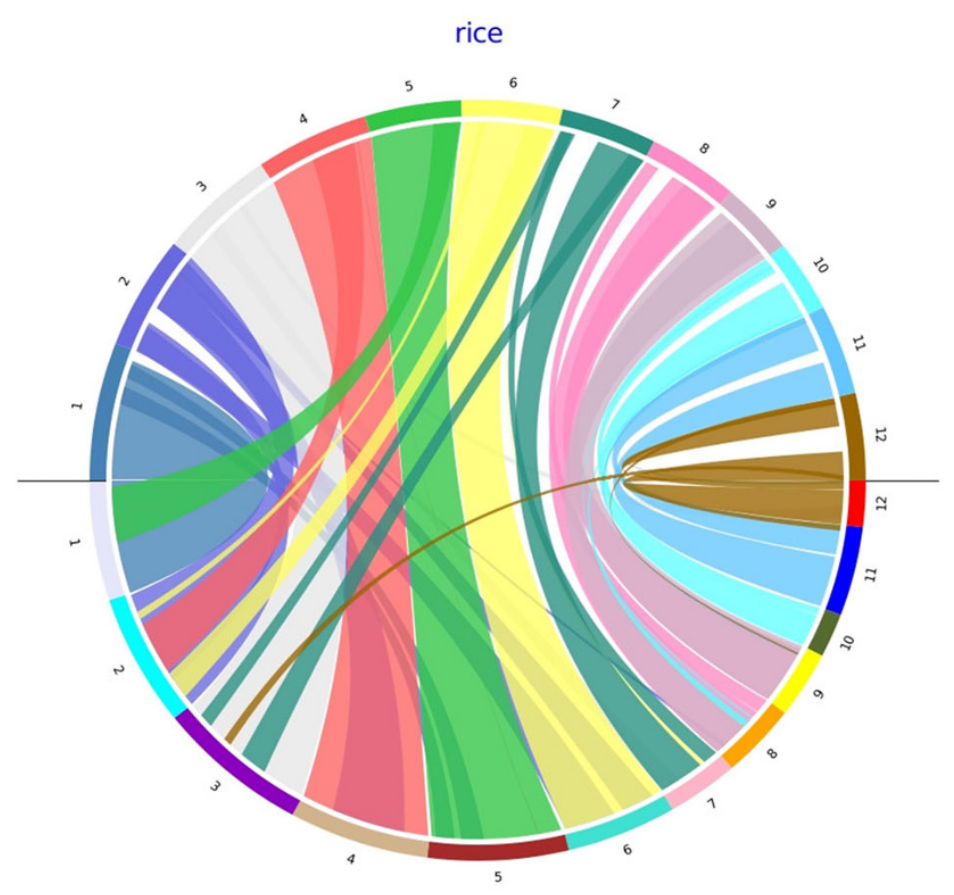

brachypodium

\section{Figure 5}

Comparative analyses of Brachypodium contigs against rice genome. Figure 5A. The SyMAP dotplot analysis of Brachypodium contig blocks against rice genome. The Brachypodium contigs were first aligned to 12 rice chromosomes based on BLAT analysis to generate 12 Brachypodium synteny contig blocks. These 12 Brachypodium synteny blocks were then compared with the pseudomolecules of rice chromosomes using dotplot analysis. Synteny blocks were detected, and background noise was filtered with SyMap [4I]. Figure 5B. Ancient duplication within the rice and Brachypodium genomes. Evidence of ancient duplication can been seen when the rice chromosome is colinear to two Brachypodium contig blocks as highlighted by colored lines in the two genomes. 
to chromosome number differences, this leaves only 19 contigs, or $5 \%$ of the total 364 contigs to be potentially due to actual or artifactual breaks in colinearity. Since there is no compelling reason why breaks in gene colinearity should coincide with gaps between BAC contigs, we therefore conclude that $B$. distachyon and rice chromosomes are highly colinear and most of the neighboring contigs on the dot-plot alignment in Figure 5A are good candidates for joining.

Most plant genomes are paleopolyploid with ancient whole-genome duplications [43-48]. The radiation of grasses was preceded by paleotetraploidy resulting in a whole-genome duplication [46], which was followed by diploidization by deletions. Therefore, in regions that are still duplicated in the B. distachyon genome, a rice region will align strongly with one $B$. distachyon contig block and weakly with another. This is evident in Figure $5 B$, where two $B$. distachyon syntenic contig blocks can be identified for several rice chromosomes. These data show that the $B$. distachyon genome has a similar set of duplications derived from the ancient paleotetraploid as does rice.

\section{Alignment of wheat EST deletion bin map to B. distachyon contigs}

The 1C nucleus of hexaploid T. aestivum contains 16,000 Mb DNA [49], which makes map-based cloning of wheat genes very difficult. Because $B$. distachyon diverged from the wheat phylogenetic lineage only about 30 million years ago (MYA) [15], B. distachyon physical map and ultimately genome sequence can facilitate wheat map-based cloning and other genomic applications.

A total of 7,104 expressed sequence tag (EST) unigenes were previously mapped into 156 deletion bins, providing a genome-wide framework for wheat mapping and identifying agronomically important genes [20]. However, a disadvantage of the deletion bin map is that loci are not ordered within the bins. Given the general colinearity among the grass genomes, this problems can be partially overcome by in silico ordering of wheat ESTs using rice genomic sequence [50]. Brachypodium is expected to show better synteny with wheat than rice because it diverged from wheat more recently than rice [3], Brachypodium is therefore expected to be more useful in comparative mapping applications than rice.

To assess the utility of $B$. distachyon physical map for wheat genomics, we compared the Brachypodium contigs with the wheat deletion bin map [20]. Of 7,104 deletion bin mapped wheat ESTs, 985 matched Brachypodium BESs at an e-value cutoff of $1 \times 10^{-10}$. These matches were derived from BESs associated with 216 contigs (32\% of total Brachypodium contigs). Such analysis allowed us to align Brachypodium contigs onto individual chromosomes based on wheat deletion bin map data http://phy map.ucdavis.edu/brachypodium/.

Comparison of Brachypodium contigs with the wheat deletion bin map and the rice genome sequence provided a genome-wide view of genome evolution among these species. Figure 6 shows an example of such a comparison. Contig138 contained 1,380 BAC clones with 1,402 BESs and spanned 2.2 $\mathrm{Mb}$ (estimated from a relationship between $\mathrm{CB}$ units and $\mathrm{Mb}$ ). A total of 16 wheat ESTs in the deletion bins matched BESs in this contig. Eight of them were mapped in the distal bins 1AS3-0.86-1.00, 1AS10.47-0.86, 1BS.sat18-0.50-1.00, 1BS.sat19-0.31.0.50, 1BS.sat19-0.31-0.50, 1BS.sat-0.31，1BS9-0.84-1.06, 1DS5-0.70-1.00, 1DS1-0.59-0.70, and 1DS3-0.48-0.59 on wheat chromosomes 1A, 1B, and 1D [20] (Figure 6). They were distributed across two to three bins on each chromosome, indicating that the contig spans multiple bins on wheat group 1 chromosomes (Figure 6). In most cases, the order of BESs in a contig homologous to wheat ESTs correlated with the order of bins along a chromosome arm into which these ESTs were mapped. These data suggested that Contig138 represented an orthologous region of these deletion bins. Alignment with rice genome indicated that Contig138 was colinear with a region on rice chromosome 5. Among eight colinear wheat ESTs between Brachypodium and wheat, three ESTs (BF484606, BF428943, and BG604768) were not colinear with the rice orthologous region. BF428943 and BG604768 were homologous to genes on rice chromosome 2, suggesting gene duplication/deletion either in rice or in the Pooideae lineage after Pooideae diverged from Ehrhartoideae but before divergence of Brachypodium. No match to BE484606 was found in the rice genome based on BLAST searches.

ESTs BF473313 and BE446475 homologous to BES sequences of Contig138 also have no match in the rice genome, but were mapped on long arms of wheat chromosomes of homeologous groups 1 and 7, respectively. These genes are only present in Brachypodium and wheat but are not located in colinear positions in the two genomes. Another EST locus, BM138418, is colinear only between Brachypodium and rice since this EST was mapped to the chromosome $3 \mathrm{~B}$ in wheat based on the wheat deletion bin map result. Two ESTs, BF484988 and BG313703, at the top of Contig138 were mapped to the short arm on wheat chromosomes of homeologous group 5. In rice, they matched sequences on chromosome 1. Other examples of noncolinear genes among the three genomes were observed. Occasional perturbations of synteny between Brachypodium, rice and wheat must therefore be expected and taken into account in comparative genomic applications. Since synteny of wheat chromosomes has been eroded faster in the distal regions than in proximal 


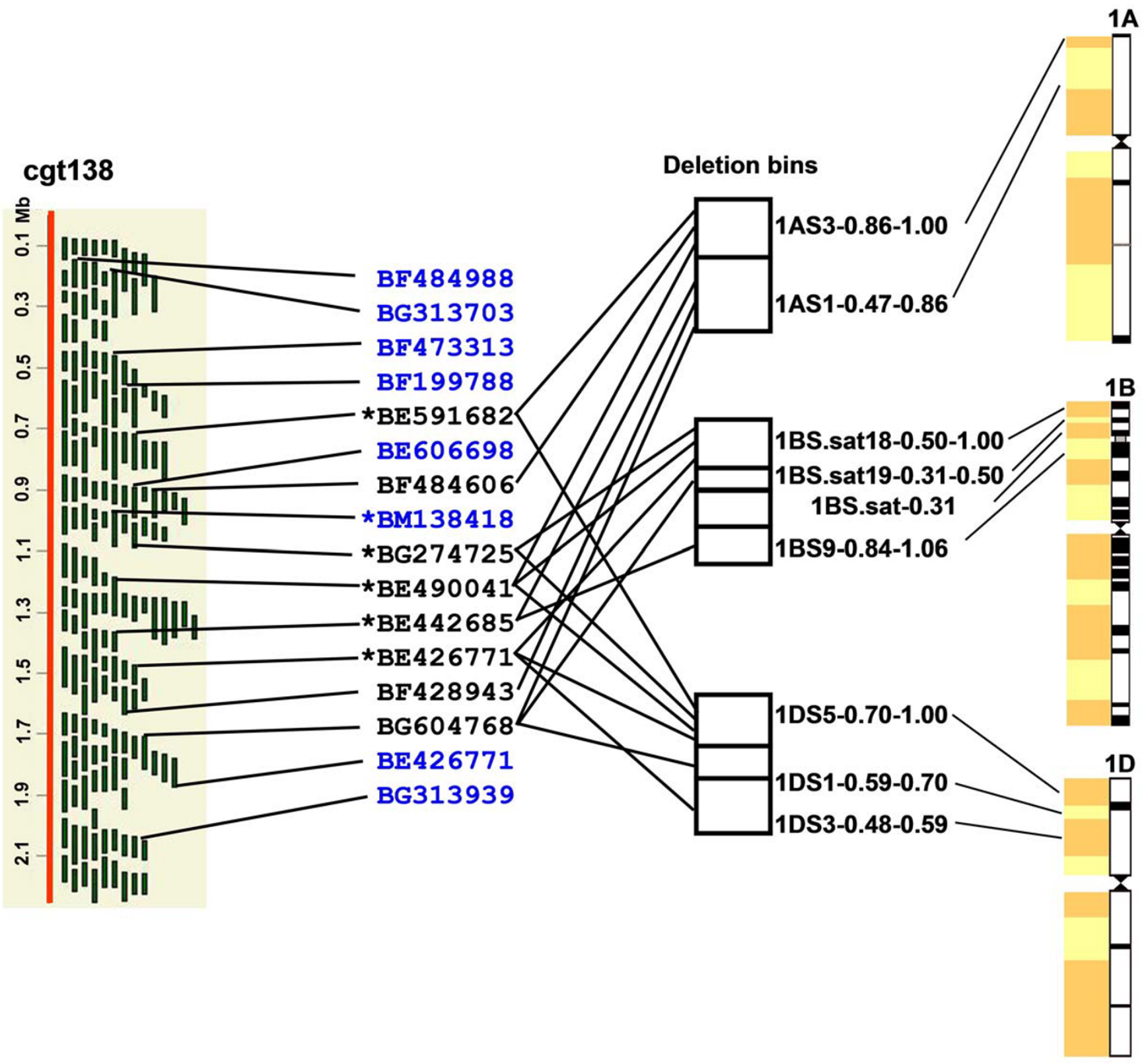

\section{Figure 6}

Anchoring Brachypodium contigs to wheat deletion bins. Brachypodium ContigI 38 is orthologous with a genomic region on rice chromosome 5 based on BLAST search. A total of 16 mapped wheat ESTs were matched by the BES in the contig. The ESTs were connected to the contig by a line from only one representative BAC end. Eight ESTs in black were mapped to deletion bins located at the distal regions of wheat group I chromosomes. The bin location for each EST was indicated by a black line. A schematic drawing of wheat group I chromosomes and associated deletion bins as indicated by colored boxes are provided to specify the fraction lengths of wheat chromosome bins. ESTs in blue represent those that were mapped to different regions in the wheat genome. Colinear ESTs between rice and Brachypodium are indicated by a star. BAC clones are indicated by a solid vertical bar in Contig /38. The approximate distance along the contig was calculated based on CB units and is indicated in Mb.

regions [51], perturbed synteny should be expected particularly in genomic comparisons with Brachypodium involving distal regions of wheat chromosomes. Synteny perturbations observed in contig 138 exemplify this real- ity since the contig is orthologous to a distal region of the short arm of chromosome 1.

To further expand the utility of the Brachypodium physical map, we integrated wheat ESTs that have not been placed 
into wheat deletion bins into Brachypodium contigs. There are over one million wheat ESTs in Genbank, and they have been assembled into 42,848 unigene sets http:// www.ncbi.nlm.nih.gov/unigene. Using BLAT, 5,421 different unigenes were integrated to the Brachypodium contigs. They were homologous to a total of 5,910 BES in the physical map. These wheat EST markers will further facilitate comparative mapping in wheat. The comparison of Brachypodium contigs with wheat deletion maps and integration of wheat unigenes into Brachypodium contigs can be accessed at http://phymap.ucdavis.edu/brachypo dium/.

\section{Conclusion}

A whole-genome, BAC-based physical map for the new grass model species, Brachypodium, was constructed. The map facilitated a variety of genomic applications. Comparison of contigs with rice revealed high colinearity between rice and Brachypodium chromosomes. Additionally, many Brachypodium BAC contigs could be anchored on the wheat deletion bin maps. Both outcomes support the anticipated utility of Brachypodium for wheat comparative genomics and practical applications in map-based cloning of wheat genes. Another application of the physical map is in the Brachypodium genome sequencing project. The phase II physical map can be aligned with shotgun sequence contigs via BES and provide scaffolds for ordering shotgun sequence contigs and estimating gap sizes. The BAC-based physical map reported here was provided to the Brachypodium distachyon sequence assembly team and has helped produce a high quality final sequence assembly http://www.brachypodium.org/node/ 18. The physical map can also identify BACs spanning sequence gaps and has served as sequencing template to fill them. Brachypodium is expected to serve as a surrogate to assist gene discovery and functional characterization in the large and complex crop genomes, such as wheat and bioenergy crops. All the data and resources developed in this study are available at the http://phymap.ucdavis.edu/ brachypodium/ website for community use.

\section{Methods \\ Bacterial artificial chromosomal (BAC) libraries}

The $B$. distachyon BAC libraries used in this study were previously constructed using partial digests with HindIII and BamHI restriction enzymes from an inbred diploid line of $B$. distachyon, $\mathrm{Bd} 21$, the same line selected for complete sequencing by the US Department of Energy Joint Genome Institute through its "Community Sequencing Program" http://www.jgi.doe.gov/sequencing/why/ 51281.html[21]. These BAC libraries represent $~ 29$.2-fold haploid genome equivalents and were employed for BAC fingerprinting and contig assembly.

\section{BAC fingerprinting and fragment sizing}

The BAC clones were fingerprinted as described by [22] with minor modifications. From each 384-well plate, four 96-well blocks containing $1.2 \mathrm{ml}$ of $2 \times$ YT medium plus $12.5 \mu \mathrm{g} / \mathrm{ml}$ chloramphenicol were inoculated with a 96well replicator. Two pins were removed from the replicator to allow the insertion of control clones into the 96well plate. Two control BAC clones were inserted manually in wells E07 and $\mathrm{H} 12$ in each 96-well block. The plates were covered with Airpore gas permeable plate sealant (Qiagen) and shaken on an orbital shaker agitated at $400 \mathrm{rpm}$ at $37^{\circ} \mathrm{C}$ for 20 hours. BAC DNA was isolated with the Qiagen R.E.A.L 96-Prep kit (Qiagen, Valencia, California). The following minor modifications of the fingerprinting method were made to accommodate the use of ABI3730XL (Applied Biosystems, Foster City, California) instead of ABI3100 for capillary electrophoresis. The more sensitive laser of the ABI3730XL instrument improved fingerprinting resolution and made it possible to reduce the amount of BAC DNA sample for electrophoresis, thus lowering fingerprinting costs. To reduce sample size, 0.5-1.2 $\mu \mathrm{g}$ instead of 1.0-2.0 $\mu \mathrm{g}$ of BAC DNA was simultaneously digested with 2.0 instead of 5.0 units each BamHI, EcoRI, XbaI, XhoI and HaeIII (New England Biolabs, Beverly, Massachusetts) at $37^{\circ} \mathrm{C}$ for $3 \mathrm{hrs}$. The DNA was labeled with $0.4 \mu \mathrm{l}$ instead of $1.0 \mu \mathrm{l}$ of the SNaPshot kit (Applied Biosystems, Foster City, California) at $65^{\circ} \mathrm{C}$ for $1 \mathrm{hr}$ and precipitated with ethanol. The labeled DNA was dissolved in $9.9 \mu \mathrm{l}$ of Hi-Di formamide, and 0.3 $\mu \mathrm{l}$ of GeneScan 1200 LIZ (Applied Biosystems, Foster City, California) was added to each sample as an internal size standard. Restriction fragments were sized with ABI3730XL using $50 \mathrm{~cm}$ capillaries and POP7 (Applied Biosystems, Foster City, California). The fragment size calling was accomplished with the GeneMaper software (Applied Biosystems, Foster City, California) with the help of FP Pipeliner http://www.bioinforsoft.com/.

\section{Fingerprints editing and contig assembly}

The fingerprint profiles for each BAC clone were collected by GeneMapper v3.7 (Applied Biosystems). The GeneMaper output data was edited with the GenoProfiler program [26]. The two control BAC clones inserted in each 96-well plate were used to check for the correct orientation of the plate. Fragments in the size range of 100 - 1,000 bp were measured. For the data quality control, vector bands and clones failing fingerprinting or lacking inserts were removed using GenoProfiler program. In addition, samples with less than 40 or more than 150 fragments were also eliminated. Fingerprints of cross-contaminated samples were detected using a module in the GenoProfiler [26] and removed from the data set. The cross-contamination was defined as clones residing in neighboring wells either in 384-well format or 96-well format (quadrants) 
share $30 \%$ or more of the mean number of fragments, the formula is shared bands*2/(bands $1+$ bands 2$)$.

A total of 52,343 BAC clones that passed quality check were used to assemble contigs using the FPC v8.5.3 [27]. The initial assembly used a Sulston score of $1 \times 10^{-45}$ and a tolerance $0.4 \mathrm{bp}$. The contig assembly was processed through the DQ function of FPC to break up contigs having more than $10 \%$ Q-clones in a contig. The assembly was further refined using "Single-to-End" and "End-toEnd" merging by stepwise decreasing of assembly stringency of Sulston score cutoff values (down to $1 \times 10^{-14}$ ).

\section{Alignment of FPC contig with the rice genome and wheat deletion bin map}

There were 64,697 BAC end sequences generated from the fingerprinted $B$. distachyon BAC clones [3] which were compared using BLAT [40] against the rice pseudomolecules build 4.0 http://rgp.dna.affrc.go.jp/IRGSP/Build4/ build4.html. The results were used as input into Synteny Mapping and Analysis Program (SyMap) [41], which computed the synteny blocks. The results can be viewed from the SyMAP Java based dot-plot, synteny block to chromosome, and close-up views.

To anchor B. distachyon physical contigs to the wheat deletion bin map, BAC-end sequence data from the B. distachyon BACs were compared against sequences of the mapped wheat EST probe sets using BLAT (parameters minScore $=50$, minIdentity $=80 \%$ ) and links to the wheat deletion bin map constructed $[20,52]$. The BLAT alignments and FPC anchoring were carried out using FPC modules BSS, which is designed for this purpose [53]. The CMAP http://gmod.org/wiki/Cmap tool allowed for comparative analysis between the $B$. distachyon physical contigs and wheat deletion bin map.

\section{Authors' contributions}

YQG, YM, NH, and JPV isolated BAC DNA. YM generated the fingerprinting data. MCL, FMY, and NH analyzed fingerprints, assembled the physical map, and verified assembly. FMY, MCL, GRL, WMN, and CS developed database and interfaces to display FPC results on the web and performed comparative studies. YQG and MCL conceived the project, supervised its execution, and draft the manuscript. JPV, JD, and ODA contributed to the planning of the project and edited the manuscript. All authors read and approved the final version of the manuscript.

\section{Acknowledgements}

The authors thank Daniel Hayden, Devin Coleman and Theresa Hill for assistance in BAC DNA extraction; Charles $\mathrm{H}$. Wang and Rana Nederi for assistance in generating BAC fingerprints. This work was supported in part by the United State Department of Agriculture, Agriculture Research Service CRIS 532502100-010, 533502100-0II, and 532502100-013; and by the Department of Plant Sciences, University of California, Davis.

\section{References}

I. IRGSP: The map-based sequence of the rice genome. Nature 2005, 436(7052):793-800.

2. Garvin DF, Gu Y-Q, Hasterok R, Hazen SP, Jenkins G, Mockler TC, Mur LAJ, Vogel JP: Development of genetic and genomic research resources for Brachypodium distachyon, a new model system for grass crop research. Crop Sci 2008, 48(Supplement_I):S-69-84.

3. Huo N, Lazo GR, Vogel JP, You FM, Ma Y, Hayden DM, ColemanDerr D, Hill TA, Dvorak J, Anderson OD, et al.: The nuclear genome of Brachypodium distachyon: analysis of BAC end sequences. Funct Integr Genomics 2008, 8(2): 135-147.

4. Hasterok R, Marasek A, Donnison IS, Armstead I, Thomas A, King IP, Wolny E, Idziak D, Draper J, Jenkins G: Alignment of the genomes of Brachypodium distachyon and temperate cereals and grasses using BAC landing with fluorescent in situ hybridization. Genetics 2006:349-362.

5. Foote TN, Griffiths S, Allouis S, Moore G: Construction and analysis of a BAC library in the grass Brachypodium sylvaticum: its use as a tool to bridge the gap between rice and wheat in elucidating gene content. Funct Integr Genomics 2004, 4(I):26-33.

6. Draper J, Mur LA, Jenkins G, Ghosh-Biswas GC, Bablak P, Hasterok $R$, Routledge AP: Brachypodium distachyon. A new model system for functional genomics in grasses. Plant Physiol 200I, I 27(4): 1539-| 555.

7. Opanowicz M, Vain P, Draper J, Parker D, Doonan JH: Brachypodium distachyon: making hay with a wild grass. Trends in Plant Science 2008, 13(4): 172-177.

8. Ozdemir BS, Hernandez P, Filiz E, Budak H: Brachypodium genomics. Int J Plant Genomics 2008, 2008:536104.

9. Vogel J, Garvin D, Leong O, Hayden D: Agrobacterium-mediated transformation and inbred line development in the model grass Brachypodium distachyon. Plant Cell, Tissue and Organ Culture 2006, 84(2): $100179-100191$.

10. Kellogg EA: Evolutionary History of the Grasses. Plant Physiol 200I, I 25(3): I I 98-I205.

II. Bennett MD, Leitch IJ: Nuclear DNA Amounts in Angiosperms: Progress, Problems and Prospects. Ann Bot 2005, 95(I):45-90.

12. Pacurar DI, Thordal-Christensen H, Nielsen KK, Lenk I: A highthroughput Agrobacterium-mediated transformation system for the grass model species Brachypodium distachyon $L$. Transgenic Res 2008, 17(5):965-975.

13. Vain P, Worland B, Thole V, McKenzie N, Alves SC, Opanowicz M, Fish LJ, Bevan MW, Snape JW: Agrobacterium-mediated transformation of the temperate grass Brachypodium distachyon (genotype Bd2I) for T-DNA insertional mutagenesis. Plant Biotechnol J 2008, 6(3):236-245.

14. Vogel J, Hill T: High-efficiency Agrobacterium-mediated transformation of Brachypodium distachyon inbred line Bd2I-3. Plant Cell Rep 2008, 27(3):47I-478.

15. Bossolini E, Wicker T, Knobel PA, Keller B: Comparison of orthologous loci from small grass genomes Brachypodium and rice: implications for wheat genomics and grass genome annotation. Plant J 2007, 49(4):704-717.

16. Faris JD, Zhang Z, Fellers JP, Gill BS: Micro-colinearity between rice, Brachypodium, and Triticum monococcum at the wheat domestication locus Q. Funct Integr Genomics 2008, 8(2): I49-164.

17. Spielmeyer W, Singh RP, McFadden H, Wellings CR, Huerta-Espino J, Kong X, Appels R, Lagudah ES: Fine scale genetic and physical mapping using interstitial deletion mutants of Lr34/YrI 8: a disease resistance locus effective against multiple pathogens in wheat. Theor Appl Genet 2008, I I 6(4):48I-490.

18. Griffiths S, Sharp R, Foote TN, Bertin I, Wanous M, Reader S, Colas I, Moore G: Molecular characterization of PhI as a major chromosome pairing locus in polyploid wheat. Nature 2006, 439(7077):749-752.

19. Huo N, Vogel JP, Lazo GR, You FM, Ma Y, McMahon S, Dvorak J, Anderson OD, Luo MC, Gu YQ: Structural characterization of Brachypodium genome and its syntenic relationship with rice and wheat. Plant Mol Biol 2009, 70(I-2):47-6I.

20. Qi LL, Echalier B, Chao S, Lazo GR, Butler GE, Anderson OD, Akhunov ED, Dvorak J, Linkiewicz AM, Ratnasiri A, et al.: A chromosome bin map of 16,000 expressed sequence tag loci and distribution of genes among the three genomes of polyploid wheat. Genetics 2004, 168(2):701-7I2. 
21. Huo N, Gu YQ, Lazo GR, Vogel JP, Coleman-Derr D, Luo MC, Thilmony R, Garvin DF, Anderson OD: Construction and characterization of two BAC libraries from Brachypodium distachyon, a new model for grass genomics. Genome 2006, 49(9): 1099-1 108.

22. Luo MC, Thomas C, You FM, Hsiao J, Ouyang S, Buell CR, Malandro $M$, McGuire PE, Anderson OD, Dvorak J: High-throughput fingerprinting of bacterial artificial chromosomes using the SNaPshot labeling kit and sizing of restriction fragments by capillary electrophoresis. Genomics 2003, 82:378-389.

23. Nelson WM, Dvorak J, Luo MC, Messing J, Wing RA, Soderlund C: Efficacy of clone fingerprinting methodologies. Genomics 2007 89(I): $160-165$.

24. Nelson WM, Bharti AK, Butler E, Wei F, Fuks G, Kim H, Wing RA, Messing J, Soderlund C: Whole-genome validation of high-information-content fingerprinting. Plant Physiol 2005, I39(I):27-38.

25. Mun JH, Kwon SJ, Yang TJ, Kim HS, Choi BS, Baek S, Kim JS, Jin M Kim JA, Lim MH, et al.: The first generation of a BAC-based physical map of Brassica rapa. BMC Genomics 2008, 9:280.

26. You FM, Luo MC, Gu YQ, Lazo GR, Deal K, Dvorak J, Anderson OD GenoProfiler: batch processing of high-throughput capillary fingerprinting data. Bioinformatics 2007, 23:240-242.

27. Soderlund C, Humphray S, Dunham I, French L: Contigs built with fingerprints, markers, and FPC V4.7. Genome Res 2000, II:934-94I.

28. Kim H, San Miguel P, Nelson W, Collura K, Wissotski M, Walling JG, Kim JP, Jackson SA, Soderlund C, Wing RA: Comparative physical mapping between Oryza sativa (AA genome type) and 0 . punctata (BB genome type). Genetics 2007, 176(I):379-390.

29. Wu C, Sun S, Nimmakayala P, Santos FA, Meksem K, Springman $R$, Ding K, Lightfoot DA, Zhang HB: A BAC- and BIBAC-based physical map of the soybean genome. Genome Res 2004, 14(2):319-326.

30. Shoemaker RC, Grant D, Olson T, Warren WC, Wing R, Yu Y, Kim $H$, Cregan P, Joseph B, Futrell-Griggs M, et al.: Microsatellite discovery from BAC end sequences and genetic mapping to anchor the soybean physical and genetic maps. Genome 2008 , 5 I (4):294-302.

31. Gardiner J, Schroeder S, Polacco ML, Sanchez-Villeda H, Fang Z, Morgante M, Landewe T, Fengler K, Useche F, Hanafey M, et al:: Anchoring 9,371 maize expressed sequence tagged unigenes to the bacterial artificial chromosome contig map by two-dimensional overgo hybridization. Plant Physiol 2004 134(4): 1317-1326.

32. Wu X, Zhong G, Findley SD, Cregan P, Stacey G, Nguyen HT: Genetic marker anchoring by six-dimensional pools for development of a soybean physical map. BMC Genomics 2008, 9:28.

33. Luo MC, Xu K, Ma Y, Deal KR, Nicolet CM, Dvorak J: A highthroughput strategy for screening of bacterial artificial chromosome libraries and anchoring of clones on a genetic map constructed with single nucleotide polymorphisms. $B M C$ Genomics 2009, 10:28.

34. Paux E, Roger D, Badaeva E, Gay G, Bernard M, Sourdille P, Feuillet $\mathrm{C}$ : Characterizing the composition and evolution of homoeologous genomes in hexaploid wheat through BAC-end sequencing on chromosome 3B. Plant J 2006, 48(3):463-474.

35. Messing J, Bharti AK, Karlowski WM, Gundlach H, Kim HR, Yu Y, Wei F, Fuks G, Soderlund CA, Mayer KFX, et al.: Sequence composition and genome organization of maize. Proc Natl Acad Sci USA 2004, I0I(40): I4349-14354.

36. Lai C, Yu Q, Hou S, Skelton R, Jones M, Lewis K, Murray J, Eustice M, Guan P, Agbayani R, et al.: Analysis of papaya BAC end sequences reveals first insights into the organization of a fruit tree genome. Molecular Genetics and Genomics 2006, 276(I): $1-12$

37. Venter JC, Smith HO, Hood L: A new strategy for genome sequencing. Nature 1996, 38 I(658I):364-366.

38. Smith DB, Flavell RB: Characterization of wheat genome by renaturation kinetics. Chromosoma 1975, 50:223-242

39. Akhunov ED, Akhunova AR, Dvorak J: BAC libraries of Triticum urartu, Aegilops speltoides and Ae. tauschii, the diploid ancestors of polyploid wheat. Theor Appl Genet 2005 III(8): 1617-1622.

40. Kent W]: BLAT--the BLAST-like alignment tool. Genome Res 2002, I 2(4):656-664.
4I. Soderlund C, Nelson W, Shoemaker A, Paterson A: SyMAP: A system for discovering and viewing syntenic regions of FPC maps. Genome Res 2006, 16(9): I I59-1168.

42. Lai J, Ma J, Swigonova Z, Ramakrishna W, Linton E, Llaca V, Tanyolac B, Park YJ, Jeong OY, Bennetzen JL, et al.: Gene loss and movement in the maize genome. Genome Res 2004 I4(I0A): | 924-1931.

43. Salse J, Bolot S, Throude M, Jouffe V, Piegu B, Quraishi UM, Calcagno $\mathrm{T}$, Cooke R, Delseny M, Feuillet C: Identification and characterization of shared duplications between rice and wheat provide new insight into grass genome evolution. Plant Cell 2008, 20(I):II-24.

44. Yu J, Wang J, Lin W, Li S, Li H, Zhou J, Ni P, Dong W, Hu S, Zeng C, et al:: The genomes of Oryza sativa: A history of duplications. PLoS Biology 2005, 3(2):e38.

45. Wei F, Coe E, Nelson W, Bharti AK, Engler F, Butler E, Kim H, Goicoechea JL, Chen M, Lee S, et al.: Physical and genetic structure of the maize genome reflects its complex evolutionary history. PLoS Genetics 2007, 3(7): el 23.

46. Paterson AH, Bowers JE, Chapman BA: Ancient polyploidization predating divergence of the cereals, and its consequences for comparative genomics. PNAS 2004, I0I(26):9903-9908.

47. Blanc G, Barakat A, Guyot R, Cooke R, Delseny M: Extensive duplication and reshuffling in the Arabidopsis genome. Plant Cell 2000, I 2(7): 1093-II0|

48. Vision TJ, Brown DG, Tanksley SD: The origins of genomic duplications in Arabidopsis. Science 2000, 290(5499):2 I I4-2 I I7.

49. Arumuganathan K, Earle ED: Nuclear DNA content of some important plant species. Plant Mol Biol Reptr 1991, 9:2II-2I5.

50. Sorrells ME, La Rota M, Bermudez-Kandianis CE, Greene RA, Kantety R, Munkvold JD, Miftahudin, Mahmoud A, Ma X, Gustafson PJ, et al.: Comparative DNA sequence analysis of wheat and rice genomes. Genome Res 2003, 13(8): 18|8-1827.

5I. Akhunov ED, Akhunov AR, Linkiewicz AM, Dubcovsky J, Hummel D, Lazo GL, Chao S, Anderson OD, David J, Qi L-L, Echalier B, et al.: Synteny pertubations between wheat homoeologous chromosomes caused by locus duplications and deletions correlate with recombination rates. Proc Natl Acad Sci USA 2004, 100: 10836-I084I.

52. Lazo GR, Chao S, Hummel DD, Edwards H, Crossman CC, Lui N, Matthews DE, Carollo VL, Hane DL, You FM, et al.: Development of an expressed sequence tag (EST) resource for wheat (Triticum aestivum L.): EST generation, unigene analysis, probe selection and bioinformatics for a 16,000-locus bindelineated map. Genetics 2004, 168(2):585-593.

53. Nelson W, Soderlund C: Integrating sequence with FPCfingerprint maps. Nucleic Acids Res 2009, 37(5):e36.

Publish with Bio Med Central and every scientist can read your work free of charge

"BioMed Central will be the most significant development for disseminating the results of biomedical research in our lifetime. "

Sir Paul Nurse, Cancer Research UK

Your research papers will be:

- available free of charge to the entire biomedical community

- peer reviewed and published immediately upon acceptance

- cited in PubMed and archived on PubMed Central

- yours - you keep the copyright
BioMedcentral 